A better result is known, ${ }^{6}$ but it is interesting to notice that this can be proved by elementary means since (5) can. ${ }^{7}$

Faculté des Sciences d'Orsay, France

\title{
SOME PROPERTIES OF INTEGRAL CLOSURE
}

\section{WILLIAM J. HEINZER}

Let $D$ be an integrally closed domain with identity having quotient field $K$, let $L$ be an algebraic extension field of $K$, and let $\bar{D}$ be the integral closure of $D$ in $L$. We prove here that the following five ideal theoretic structure properties of $\bar{D}$ are inherited by $D$, namely: (a) $\bar{D}$ is a Prüfer domain, (b) $\bar{D}$ is an almost Dedekind domain, ${ }^{1}$ (c) $\bar{D}$ is a Dedekind domain, (d) $\bar{D}$ has the $Q R$-property, ${ }^{2}$ (e) $\bar{D}$ has property (\#). ${ }^{3}$

The converse of (a) is true (that is, $D$ Prüfer implies that $\bar{D}$ is Prüfer) and was established by Prüfer in $[11$, p. 31]. In case $L$ is finite-dimensional over $K$, Noether [9, p. 37] proved the converse of (c) and Butts and Phillips [2, p. 270] proved the converse of (b). In the general case it is well-known or easy to see that the converses of (b), (c) and (e) are false. The converse of (d) is false (see [6, p. 102]) even when $L$ is finite-dimensional over $K$.

Our statements concerning (a), (b) and (c) will be obtained as corollaries to the following.

Theorem 1. Let $M$ be a prime ideal of $\bar{D}$ and let $P=M \cap D$. Then $\bar{D}_{M} \cap K=D_{P}$.

Proof. It is clear that $D_{P}$ is contained in $\bar{D}_{M} \cap K$. To obtain the reverse inclusion we first observe that $L$ may be assumed to be a normal extension of $K$. For let $E$ be a normal closure of $L$ over $K, D^{*}$ the integral closure of $\bar{D}$ in $E$, and $N$ a prime ideal of $D^{*}$ lying over $M$. Then $N \cap D=P$ and $\bar{D}_{M} \subseteq D_{N}^{*} \cap L$ so that $\bar{D}_{M} \cap K \subseteq D_{N}^{*} \cap K$. Let $\left\{M_{\alpha}\right\}$ be the set of prime ideals of $\bar{D}$ lying over $P$. By a well-known

Received by the editors July 26, 1966.

${ }^{1} D$ is almost Dedekind if for each maximal ideal $P$ of $D, D_{P}$ is a discrete rank one valuation ring $[4$, p. 813$]$.

${ }^{2} D$ has the $Q R$-property if each integral domain between $D$ and its quotient field is a quotient ring of $D[6$, p. 97].

${ }^{3} D$ is said to have property (\#) if for $\Delta_{1}$ and $\Delta_{2}$ distinct subsets of the set of maximal ideals of $\bar{D}$ we have $\bigcap_{M \in \Delta_{1}} D_{M} \neq \bigcap_{M \in \Delta_{2}} D_{M}[5$, p. 331]. 
theorem due originally to Krull $[7, \mathrm{p} .752]$ the $M_{\alpha}$ 's are conjugate over $K$, that is, for each $\alpha$ there is an automorphism $\sigma_{\alpha}$ of $L$ over $K$ such that $\sigma_{\alpha}(M)=M_{\alpha}$. It follows that $\sigma_{\alpha}\left(\bar{D}_{M}\right)=\bar{D}_{M_{\alpha}}$. Hence $\bar{D}_{M} \cap K$ $=\bar{D}_{M_{\alpha}} \cap K=\bigcap_{\alpha}\left(\bar{D}_{M_{\alpha}} \cap K\right)$. If $S=D-P$ then $\bar{D}_{S}$ is integral over $D_{S}$ so that $\bar{D}_{S} \cap K=D_{S}$. Moreover, $\left\{M_{\alpha}\right\}$ is the set of ideals of $\bar{D}$ maximal with respect to not meeting $S[8, \mathrm{p} .30]$. Hence $\left\{M_{\alpha} \bar{D}_{S}\right\}$ is the set of maximal ideals of $\bar{D}_{S}$. Since $\bar{D}_{M_{\alpha}}=\left(\bar{D}_{S}\right)_{M_{\alpha}} \bar{D}_{S}$ for each $\alpha$, we have $\bar{D}_{S}=\bigcap_{\alpha}\left(\bar{D}_{S}\right)_{M_{\alpha}} \bar{D}_{S_{\alpha}}=\bigcap_{\alpha} \bar{D}_{M_{\alpha}}$. Therefore, $\bar{D}_{M} \cap K=\bigcap_{\alpha}\left(\bar{D}_{M_{\alpha}} \cap K\right)=$ $\left(\bigcap_{\alpha} \bar{D}_{M_{\alpha}}\right) \cap K=\bar{D}_{S} \cap K=D_{S}$, as we wished to show.

Corollary 1. With notation as in Theorem 1 if $\bar{D}_{M}$ is a valuation ring, then $D_{P}$ is a valuation ring.

Corollary 2. If $\bar{D}_{M}$ is a valuation ring, then $\bar{D}_{M_{\alpha}}$ is a valuation ring for each prime ideal $M_{\alpha}$ of $\bar{D}$ which lies over $P$.

Proof. ${ }^{4}$ By Corollary $1, D_{P}$ is a valuation ring. If $S=D-P$, then $\bar{D}_{S}$ is the integral closure of $D_{S}$ in $L$ so that $\bar{D}_{S}$ is a Prüfer domain. $\bar{D}_{M_{\alpha}}$ is a quasi-local overring of $\bar{D}_{S}$ and thus a valuation ring.

Corollary 3. If $\bar{D}$ is a Prüfer domain, then $D$ is a Prüfer domain.

Corollary 4. If $\bar{D}$ is an almost Dedekind domain, then $D$ is almost Dedekind.

Proof. Let $P$ be a maximal ideal of $D$ and let $M$ be a maximal ideal of $\bar{D}$ lying over $P$. Since $\bar{D}$ is almost Dedekind, $\bar{D}_{M}$ is a rank one discrete valuation ring. By Theorem $1, \bar{D}_{M} \cap K=D_{P}$ so that $D_{P}$ is also a rank one discrete valuation ring. Hence $D$ is almost Dedekind.

Corollary 5. If $\bar{D}$ is a Dedekind domain, then $D$ is Dedekind.

Proof. ${ }^{5} \bar{D}$ Dedekind implies, using Corollary 4 , that $D$ is an almost Dedekind domain in which each nonzero element is contained in only finitely many maximal ideals. Hence by $[4, \mathrm{p} .815] D$ is a Dedekind domain.

A domain which has the $Q R$-property is necessarily Prüfer. Pendleton in $[10$, p. 500] has shown that a Prüfer domain $D$ has the $Q R$ property if and only if the radical of each finitely generated ideal of $D$ is the radical of a principal ideal. We use this characterization of domains with the $Q R$-property to establish the following.

4 If $L$ is normal over $K$, then the proof of Corollary 2 is immediate; for $\bar{D}_{M_{\alpha}}$ is then the image of $\bar{D}_{M}$ under a $K$-automorphism of $L$.

${ }^{b}$ Corollary 5 can also be obtained from the fact that an integral domain with identity is Dedekind if and only if it is a Krull domain of dimension $\leqq 1$. 
Theorem 2. If $\bar{D}$ is a domain with the $Q R$-property, then $D$ also has the $Q R$-property.

Proof. Since $\bar{D}$ has the $Q R$-property, $\bar{D}$ is a Prüfer domain. Hence by Corollary $3, D$ is a Prüfer domain. Let $A$ be a finitely generated ideal of $D$. We show that there is some $a \in D$ such that $\sqrt{ } A=\sqrt{ }(a)$. Denote by $\left\{P_{\alpha}\right\}$ the set of minimal primes of $A$ in $D$, and by $\left\{M_{\beta}\right\}$ the set of prime ideals of $\bar{D}$ which lie over the $P_{\alpha}$ 's. It follows from the "going down" theorem (see $\left[1\right.$, p. 255] or $\left[7\right.$, p. 755]) that $\left\{M_{\beta}\right\}$ is the set of minimal primes of $A \bar{D}$. Since $\bar{D}$ has the $Q R$-property and $A \bar{D}$ is finitely generated, there exists $a_{1} \in \bar{D}$ such that $\sqrt{ }\left(a_{1}\right)=\sqrt{ } A \bar{D}$ $=\bigcap_{\beta} M_{\beta}$. Hence $\left(a_{1}\right)$ and $A \bar{D}$ are contained in the same prime ideals of $\bar{D}$. Thus $\left\{M_{\beta}\right\}$ is the set of minimal primes of $\left(a_{1}\right)$. Let $E$ be a normal closure of $L$ over $K$, and let $a_{1}, a_{2}, \cdots, a_{8}$ be the distinct conjugates of $a_{1}$ in $E$. Denote by $\sigma_{i}$ a relative isomorphism of $L$ over $K$ in to $E$ such that $\sigma_{i}\left(a_{1}\right)=a_{i}$. Let $\sigma_{i}(L)=L_{i}$ and $\sigma_{i}(\bar{D})=\bar{D}_{i}$. Since $\sigma_{i}$ is the identity on $D$, each $\bar{D}_{i}$ is an integral extension of $D$ and $\left\{\sigma_{i}\left(M_{\beta}\right)\right\}$ is the set of prime ideals of $\bar{D}_{i}$ which lie over $\left\{P_{\alpha}\right\}$. And because $\left\{M_{\beta}\right\}$ is the set of minimal primes of $\left(a_{1}\right)$ in $\bar{D},\left\{\sigma_{i}\left(M_{\beta}\right)\right\}$ is the set of minimal primes of $\left(a_{i}\right)$ in $\bar{D}_{i}$. Denote by $p$ the characteristic of $K$ if $K$ has nonzero characteristic, $p=1$ if $K$ is of characteristic zero. We can choose a positive integer $e$ such that $a=\left(a_{1} a_{2} \cdots a_{8}\right)^{p^{e}}$ $\in K$. Then $a \in K$ and $a$ integral over $D$ imply $a \in D$. We show that $\left\{P_{\alpha}\right\}$ is the set of minimal primes of $a D$.

Let $D^{*}$ be the integral closure of $D$ in $E$. $D^{*}$ is the integral closure of $\bar{D}_{i}$ in $E$ for each $i$, and $D^{*} \cap L_{i}=\bar{D}_{i}$. If $\left\{N_{\gamma}\right\}$ is the set of primes of $D^{*}$ which lie over the $P_{\alpha}$ 's, then $N_{\gamma} \cap \bar{D}_{i}$ is a prime ideal of $\bar{D}_{i}$ which lies over some $P_{\alpha}$. Hence there is an $M_{\beta}$ such that $N_{\gamma} \cap \bar{D}_{i}=\sigma_{i}\left(M_{\beta}\right)$. Also $D^{*}$ integral over $\bar{D}_{i}$ implies that for each $\sigma_{i}\left(M_{\beta}\right)$ there is a prime ideal $N$ of $D^{*}$ lying over $\sigma_{i}\left(M_{\beta}\right)$ and $N \cap D=\sigma_{i}\left(M_{\beta}\right) \cap D=P_{\alpha}$ for some $\alpha$. Thus $N \in\left\{N_{\gamma}\right\}$. Because $\left\{\sigma_{i}\left(M_{\beta}\right)\right\}$ is the set of minimal primes of $a_{i} \bar{D}_{i},\left\{N_{\gamma}\right\}$ is the set of minimal primes of $a_{i} D^{*}, i=1,2, \cdots, s$. Hence $\left\{N_{\gamma}\right\}$ is the set of minimal primes of $a D^{*}=\left(a_{1} a_{2} \cdots a_{8}\right)^{p^{e}} D^{*}$. Since $D^{*} \cap K=D$ and $a \in D$, we have $a D^{*} \cap D=a D$. It follows that $\left\{N_{\gamma} \cap D\right\}=\left\{P_{\alpha}\right\}$ is the set of minimal primes of $a D^{*} \cap D=a D$. We have established that $\sqrt{ } A=\bigcap_{\alpha} P_{\alpha}=\sqrt{ } a D$. Hence the radical of each finitely generated ideal of $D$ is the radical of a principal ideal. Therefore $D$ has the $Q R$-property.

We remark that because the $Q R$-property is not preserved under integral extension, the proof of Theorem 2 can not be reduced to the case when $L$ is normal over $K$.

Theorem 3. If $\bar{D}$ has property (\#), then $D$ has property (\#). 
Proof. We assume that $D$ does not have property (\#). Then as observed in $\left[5\right.$, p. 331] there is a proper subset $\left\{P_{\alpha}\right\}$ of the set of maximal ideals of $D$ such that $D=\bigcap_{\alpha} D_{P_{\alpha}}$. Let $\left\{M_{\beta}\right\}$ be the set of prime ideals of $\bar{D}$ lying over the $P_{\alpha}$ 's. Since $\bar{D}$ is integral over $D$, $\left\{M_{\beta}\right\}$ is a proper subset of the set of maximal ideals of $\bar{D}$. We show that $\bar{D}=\bigcap_{\beta} \bar{D}_{M_{\beta}}$ and hence that $\bar{D}$ does not have property (\#). Let $u \in L-\bar{D}$ and let $f(X) \in K[X]$ be the minimal polynomial for $u$ over $K$. Since $u$ is not integral over $D, f(X) \notin D[X]$. Because $D=\bigcap_{\alpha} D_{P_{\alpha}}$, there is a $P \in\left\{P_{\alpha}\right\}$ such that $f(X) \notin D_{P}[X]$. If $S=D-P$, then $\bar{D}_{S}$ is the in tegral closure of $D_{S}$ in $L$ and $f(X) \notin D_{S}[X]$ implies that $u$ is not integral over $D_{S}$. Hence $u \notin \bar{D}_{S}$. If $\left\{M_{\gamma}\right\}$ is the set of prime ideals of $\bar{D}$ which lie over $P$, then $\left\{M_{\gamma}\right\} \subseteq\left\{M_{\beta}\right\}$ and the maximal ideals of $\bar{D}_{S}$ are of the form $M_{\gamma} \bar{D}_{S}$. We have $\bar{D}_{S}=\bigcap_{\gamma}\left(\bar{D}_{S}\right)_{M \gamma} \bar{D}_{S}=\bigcap_{\gamma} \bar{D}_{M \gamma}$. There-

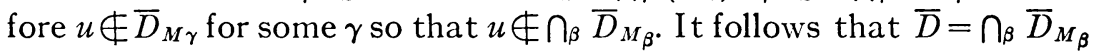
which completes the proof of Theorem 3.

If $J$ is an integral domain having quotient field $F$, we call any domain between $J$ and $F$ an overring of $J$. Using Theorem 3 and the fact that an integral domain $J$ is Prüfer if and only if each overring of $J$ is integrally closed [3, p. 198] we obtain the following corollary.

CoRollary 6. If every overring of $\bar{D}$ has property (\#), then every integrally closed overring of $D$ has property (\#). In particular, if $\bar{D}$ is a Prüfer domain and every overring of $\bar{D}$ has property (\#), then every overring of $D$ has property (\#).

In connection with Corollary 6 the following question arises: If $J^{*}$, the integral closure of a domain $J$ has property (\#), must $J$ have property (\#)? We have been unable to answer this question.

Acknowledgement. This paper is part of the author's doctoral thesis written under the direction of R. W. Gilmer, Jr. The author wishes to thank Professor Gilmer for his help and encouragement.

\section{REFERENCES}

1. I. S. Cohen and A. Seidenberg, Prime ideals and integral dependence, Bull. Amer. Math. Soc. 52 (1946), 252-261.

2. H. S. Butts and R. C. Phillips, Almost multiplication rings, Canad. J. Math. 17 (1965), 267-277.

3. E. D. Davis, Overrings of commutative rings. II, Trans. Amer. Math. Soc. 110 (1964), 196-212.

4. R. W. Gilmer, Jr., Integral domains which are almost Dedekind, Proc. Amer. Math. Soc. 15 (1964), 813-818.

5. - On overrings of Prüfer domains, J. of Algebra 4 (1966), 331-340.

6. R. W. Gilmer, Jr. and J. E. Ohm, Integral domains with quotient overrings, Math. Ann. 153 (1964), 97-103. 
7. W. Krull, Beiträge zur Arithemetik Kommutativer Integritätsbereiche. III, Math. Z. 42 (1937), 745-766.

8. M. Nagata, Local rings, Interscience, New York, 1962.

9. E. Noether, Abstracter Aufbau der Idealtheorie in algebraischen Zuhl-und Functionenkörpern, Math. Ann. 96 (1926), 26-61.

10. R. L. Pendleton, A characterization of Q-domains, Bull. Amer. Math. Soc. 72 (1966), 499-500.

11. H. Prüfer, Untersuchungen über die Teilbarkeitseigenschaften in Körpern, J. Reine Angew. Math. 168 (1932), 1-36.

12. O. Zariski and P. Samuel, Commutative algebra, Vol. I, Van Nostrand, Princeton, N. J., 1958.

13. —, Commutative algebra, Vol. II, Van Nostrand, Princeton, N. J., 1960.

Florida State University and

Louisiana State UnIVERsity 